\title{
Strut chordal-sparing mitral valve replacement preserves long-term left ventricular shape and function in pigs
}

\author{
Morten Smerup, MD, a,b Jonas Funder, MS, ${ }^{a, b}$ Camilla Nyboe, MS, ${ }^{a, b}$ Christian Høyer, MS, ${ }^{a, b}$ Troels F. Pedersen, MS, ${ }^{a, b}$ \\ Lars Ribe, BMSc, ${ }^{\mathrm{c}}$ Steffen Ringgaard, BMSc, PhD, ${ }^{\mathrm{C}}$ Won Yong Kim, MD, DMSc, ${ }^{\mathrm{c}}$ Erik M. Pedersen, MD, DMSc, ${ }^{\mathrm{C}}$ \\ Niels T. Andersen, BSc, PhD, ${ }^{d}$ Sten L. Nielsen, MD, PhD, ${ }^{a, b}$ and J. Michael Hasenkam, MD, DMSc ${ }^{a, b}$
}

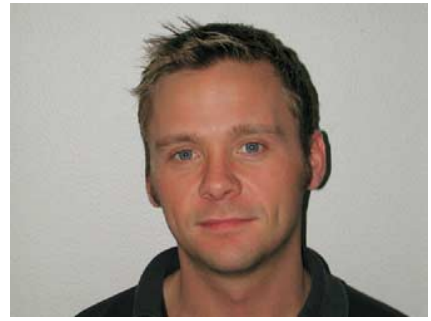

Dr Smerup
From the Department of Cardiothoracic and Vascular Surgery T, ${ }^{\mathrm{a}}$ Institute of Clinical Medicine, ${ }^{\mathrm{b}}$ The Magnetic Resonance Centre, ${ }^{\mathrm{c}}$ Aarhus University Hospital, Skejby Sygehus, Brendstrupgaardsvej, and Department of Biostatistics, Institute of Public Health, ${ }^{\mathrm{d}}$ Faculty of Health, Aarhus University, Aarhus, Denmark.

The Danish Heart Foundation, the Aase and Einar Danielsens Foundation, and the Augustinus Foundation funded this study. Mechanical heart valves were provided by St Jude Medical. The sponsors had no power of decision in terms of study design, collection, analysis, and data interpretation; manuscript drafting; and decision of submission.

Received for publication March 6, 2005; revisions received June 23, 2005; accepted for publication July 11, 2005.

Address for reprints: Morten Smerup, MD, Department of Cardiothoracic and Vascular Surgery $\mathrm{T}$ and Institute of Clinical Medicine, Aarhus University Hospital, Skejby Sygehus, Brendstrupgaardsvej, 8200 Aarhus N, Denmark (E-mail: morten.smerup@ ki.au.dk).

J Thorac Cardiovasc Surg 2005;130:1675-82 0022-5223/\$30.00

Copyright (C) 2005 by The American Association for Thoracic Surgery

doi:10.1016/j.jtcvs.2005.07.042
Objective: Mitral valve replacement with preservation of the entire subvalvular apparatus entails superior postoperative left ventricular function compared with other techniques. However, this option is often not possible because of valve pathology. We hypothesized that preservation of only 4 mitral valve secondary ("strut") chordae would be functionally and geometrically equivalent to total valve preservation in the long-term setting. In a porcine mitral valve replacement model we investigated the long-term effects of 3 surgical techniques on left ventricular function and geometry: (1) total preservation of the native valve, (2) strut chordal preservation, and (3) total excision of the subvalvular apparatus.

Methods: Forty $60-\mathrm{kg}$ pigs were randomized to 1 of the 3 techniques. Global and regional left ventricular function and dimensions were assessed with cardiovascular magnetic resonance and conductance catheter 90 days after mitral valve replacement. Groups were compared by multivariate analysis of variance.

Results: There was no overall difference between groups 1 and 2. Group 3 animals had (1) greater base-apex diastolic and systolic lengths, and smaller short-axis diameters, and (2) lower sphericity indices, and greater base-apex and short-axis fractional shortening than groups 1 and 2. Regional analysis showed slimming and elongation to occur primarily in the basal left ventricular segments. Left ventricular contractility and hemodynamic parameters did not differ between groups.

Conclusions: Strut chordal preservation was equivalent to total valve preservation during mitral valve replacement, whereas total chordal resection caused significant left ventricular slimming with compensatory increases in fractional shortening. Therefore, to preserve left ventricular geometry, special attention must be paid to maintain the valvular-ventricular continuity through the strut chordae during mitral valve replacement. This concept may have important therapeutic implications for chordal-sparing mitral valve replacement.

$\mathrm{M}$ itral valve replacement (MVR) with preservation of subvalvular structures entails superior postoperative function of the left ventricle (LV) compared with techniques in which the native valve is excised. The beneficial hemodynamic effects have been ascribed to maintain valvular-ventricular continuity in the LV and have been verified by both clinical ${ }^{1,2}$ and experimental studies. ${ }^{3,4}$ In a recent prospective randomized clinical study, ${ }^{5}$ Yun and colleagues furthermore showed that in terms of LV chamber size, systolic afterload, and ejection fraction, complete preservation of the native mitral valve during MVR is superior to preservation of only the posterior leaflet chords. However, complete preservation is often hindered by pathologic processes in the native valves such as leaflet redundancy, chordal rupture, calcification, infection, or necrotic tissue. Furthermore, because of the possible pitfalls of total preservation (interference with 


$$
\begin{aligned}
& \text { Abbreviations and Acronyms } \\
& \begin{aligned}
\mathrm{CMR} & =\text { cardiovascular magnetic resonance } \\
\mathrm{EDV} & =\text { end-diastolic volume } \\
\mathrm{ESV} & =\text { end-systolic volume } \\
\mathrm{LV} & =\text { left ventricle } \\
\mathrm{MANOVA} & =\text { multivariate analysis of variance } \\
\mathrm{MVR} & =\text { mitral valve replacement }
\end{aligned}
\end{aligned}
$$

prosthetic valve superstructures, LV outflow obstruction, and technical complexity), many surgeons often resign to preserve only parts of the subvalvular apparatus. However, lack of knowledge of the specific functional role of the anterior and posterior leaflets and their subvalvular continuity to the LV wall may result in suboptimal chordalsparing techniques.

One potential surgical alternative of preserved valvularventricular continuity is suggested by the functional anatomy of healthy mitral valves. The valve leaflets are anchored in the LV by the chordae tendineae and the anterolateral and posteromedial papillary muscles (Figure 1). Chordae can be divided into primary and secondary with respect to their insertion on the free edges of the leaflets or on the ventricular surfaces, respectively. ${ }^{6,7}$ Two particularly thick secondary chordae, the so-called strut chordae, attach each papillary muscle to the central parts of the anterior leaflets. These chordae are favorably oriented parallel to the LV long axis (compare Figure 1) and may hereby facilitate force transmission between the LV base and apex and therefore effectuate LV piston-pump function during ejection.

Accordingly, several experimental studies ${ }^{8-11}$ have suggested that these secondary (strut) chordae are pivotal for preservation of LV geometry and systolic function. Therefore, from a functional viewpoint LV function may benefit from preservation of at least the strut chordae during MVR.

In the present study we hypothesized that LV function and geometry would be preserved equally well after either total chordal preservation or preservation of the strut chordae only. Our aim was to compare these methods in a recently developed long-term porcine model for MVR. ${ }^{12}$

\section{Materials and Methods \\ Animals}

We randomized a total of 40 mixed Danish Landrace/Yorkshire female pigs (body weight $57.9 \pm 2.7 \mathrm{~kg}$ ) to MVR with (1) total preservation of the native valve, (2) preservation of only the secondary chordae (typically 2 anterior struts and 4 to 6 large posterior secondary chordae) by excision of leaflet coaptation zones and primary chordae, or (3) total excision of the native mitral valve. The same number and race of pigs (body weight $\sim 40$ $\mathrm{kg}$ ) were used as blood donors. Animal handling was performed according to the guidelines given by the Danish Inspectorate for Animal Experimentation and after specific approval from this

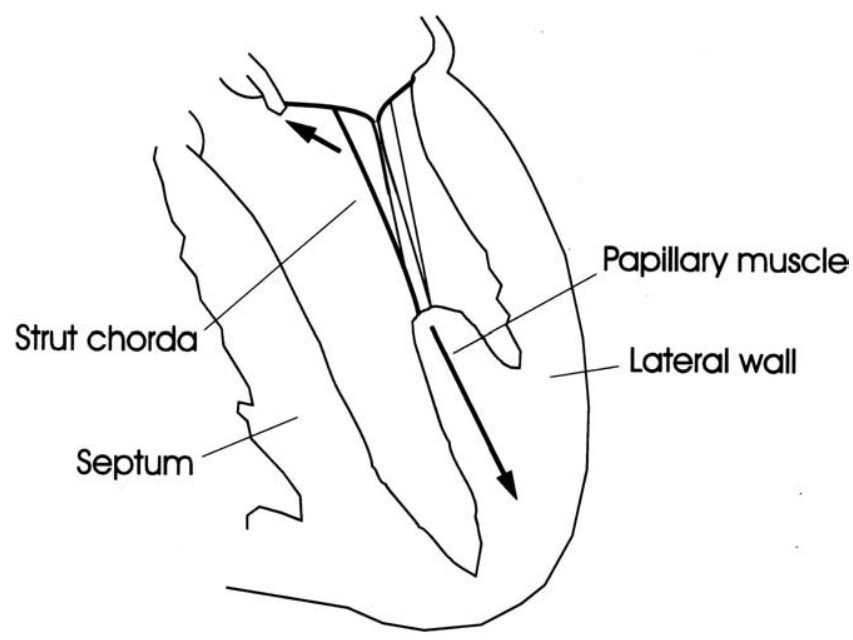

Figure 1. Left ventricular long-axis view. The secondary (strut) chordae are placed centrally within the subvalvular apparatus to ensure force transmission and geometric stability of the left ventricle (only 1 papillary muscle is shown).

institution. The veterinary technicians maintained close contact with the study leader during the postoperative period, and any signs of pain, infections, or heart failure (fatigue, dyspnea, and coughing) were immediately reported and dealt with.

\section{Mitral Valve Replacement Experiments}

We previously described these methods in detail ${ }^{12}$; therefore only relevant features will be mentioned here. The animals were randomized by an Internet-based block randomization procedure to 1 of the 3 procedures. On cardiopulmonary bypass the mitral valve was exposed through a left atriotomy. In groups 1 and 2 the redundant leaflet tissue was plicated by means of the valve sutures to avoid impingement with the prosthetic valve and thereby cause LV outflow tract obstruction. The valve (size $29 \mathrm{~mm}$, St Jude Medical, St Paul, Minn, in all cases) was hereafter secured in an anti-anatomic position by means of everted horizontal mattress sutures (double-armed 2-0 Ti-Cron 3323-56, Davis \& Geck, Danbury, Conn). Unhindered mechanical valve leaflet motion was ensured after each implantation. The animal was thereafter gently weaned from cardiopulmonary bypass and closed in the conventional manner. Postoperative analgesic and antibiotic therapy have been described in detail. ${ }^{12}$ All animals recovered in the experimental animal cardiac intensive care unit and were transported to their farming facilities approximately 24 hours after surgery for continuous observation for 5 days postoperatively. Anticoagulation was maintained by daily subcutaneous injection of $10,000 \mathrm{IU}$ of unfractionated heparin.

\section{Follow-up Examinations}

Animals were examined with cardiovascular magnetic resonance (CMR) and conductance catheter at 90 days postoperatively for assessment of global and regional function. Sedation was accomplished as mentioned previously. After measurements, animals were killed and hearts were excised and examined for evidence of any pathologic processes that would perturb mechanical valve function (eg, endocarditis, pannus, and thrombus). 


\section{Data Acquisition and Analysis}

\section{Global left ventricular function and geometry}

CARDIOVASCULAR MAGNETIC RESONANCE MEASUREMENTS. All CMR examinations were performed with a Philips Intera $1.5 \mathrm{~T}$ magnetic resonance whole-body scanner (Phillips, Best, The Netherlands), equipped with PowerTrack 6000 Gradients and Software Release 9.1.1. A fluid-filled catheter was introduced through the carotid artery for measurement of aortic and LV pressures.

BASIC HEMODYNAMIC (FUNCTIONAL) DATA. By following scout images to determine the position and orientation of the LV long-axis, conventional 2-chamber, 4-chamber, and long-axis views were acquired during end-expiratory apnea using a prospective, electrocardiogram-triggered steady-state free precession breath-hold cine sequence. ${ }^{13}$ A stack of 6 -mm thick contiguous slices encompassing the LV from base to apex in the cardiac short-axis orientation was acquired for volumetric measurements. Imaging parameters included the following: repetition time $=3.0$ $\mathrm{ms}$, echo time $=1.49 \mathrm{~ms}$, flip angle $=65^{\circ}$, acquisition matrix $=$ $160 \times 146$, field of view $=320 \times 320 \mathrm{~mm}^{2}$, half Fourier acquisition in the phase encoding direction, spatial in-plane resolution $=2.0 \times 2.19 \mathrm{~mm}$, and slice thickness $=6 \mathrm{~mm}$. Enddiastolic volume (EDV), end-systolic volume (ESV), left ventricular mass, stroke volume, cardiac output, and ejection fraction were calculated by segmentation of end-diastolic and end-systolic areas. ${ }^{14} \mathrm{LV}$ pressure was measured immediately after magnetic resonance examinations with a Micro-Tip pressure catheter (Millar Instruments, Inc, Houston, Tex). This value was averaged over 20 heart cycles.

BASE-APEX AND SHORT-AXIS LINEAR DIMENSIONS. A mean base-apex length in end-diastole and end-systole were calculated from the 2-chamber, 4-chamber, and long-axis views as the distance from the LV apex to the center as well as the edges of the mechanical valve sewing ring. A mean short-axis diameter in end-diastole and end-systole was calculated from the septal-lateral and anterior-posterior diameters measured from mid-cavity shortaxis views. Fractional shortenings of base-apex lengths and shortaxis diameters were calculated as:

$$
F S=\frac{\text { End-diastolic length }- \text { End-systolic length }}{\text { End-diastolic length }}
$$

LV diastolic and systolic sphericity indices (SI-dia and SI-sys) and base-apex/short-axis indices (AI-dia and AI-sys) were calculated from EDV, ESV, and 4-chamber diastolic and systolic measures as follows ${ }^{15,16}$ :

$$
\begin{gathered}
S I=\frac{V}{\left(4 / 3 \times \pi \times(L / 2)^{3}\right)} \\
A I=\frac{(L / 2)}{\sqrt{\frac{3 \times V}{4 \times \pi \times(L / 2)}}}
\end{gathered}
$$

where $\mathrm{V}$ is EDV or ESV and L is mean base-apex length.

\section{Conductance Catheter Measurements}

Immediately after CMR measurements a conductance catheter (SPC-570 7F, Millar Instruments) was placed in the LV for as- sessment of indices of contractility. ${ }^{17}$ Segmental conductance signals (5 channels) and LV pressure were sampled by a signal conditioner (Sigma 5 DF, Leycom, Leyden, The Netherlands) and stored on a personal computer with custom-made software. To achieve a constant heart rate, right atrial pacing (100 bpm) was instituted. The conductance signal was calibrated as follows: Instantaneous LV volume $\mathrm{V}$ can be calculated from the total measured conductance $\mathrm{G}$ from the linear relationship:

$$
V=\alpha^{\prime}(G-P W C)
$$

where $\alpha^{\prime}$ is a scalar and PWC is the total parallel wall conductance (assumed to be constant throughout the cardiac cycle and during preload reduction). EDV, ESV, and ejection fraction are calculated from CMR measurements and assumed to be constant (without pacing). Therefore $\alpha^{\prime}$ and PWC can be calculated as:

$$
\begin{gathered}
P W C=G_{E D V}-\left(\frac{G_{E D V}-F_{E S V}}{E F}\right) \\
\alpha^{\prime}=\frac{E D V-E S V}{G_{E D V}-G_{E S V}}
\end{gathered}
$$

where $G_{E D V}$ and $G_{E S v}$ are the end-diastolic and end-systolic conductances, respectively.

Maximum rate of aortic pressure increase $(\mathrm{dP} / \mathrm{dt}$ max) was averaged over 20 consecutive heart cycles. Preload independent parameters of contractility were sampled during internal balloon occlusion $(1.4 \mathrm{~cm} 7 \mathrm{~F}$ embolectomy catheter, Sorin Biomedical, Irvine, Calif) of the inferior vena cava: End-systolic elastance was calculated as the slope of the end-systolic pressure-volume relationship, and preload recruitable stroke work was calculated as the slope of the stroke work-EDV relation with dedicated software (PVAN 2.9, Millar Instruments).

\section{Regional Left Ventricular Function and Geometry}

For assessment of regional LV function and geometry, CMR short-axis slices were examined for end-diastolic area, end-systolic area, and myocardial thickening (in percentage of end-diastolic wall area). Slices were aligned from apex to basis as follows: The most apical slice with both an end-diastolic and an end-systolic area was defined as number 1, the next most apical as number 2, and so forth. Easyvision (Phillips) software was used for data analysis.

\section{Statistical Analysis}

The number and causes of deaths between groups were analyzed with the Fisher exact test. All other results are described as mean \pm standard deviation with 95\% confidence intervals where appropriate. Multivariate analysis of variance (MANOVA) testing of groups of parameters (eg, basic hemodynamics, lengths, indices, and LV-contractility indices) of all groups was performed first at a significance level of 5\%. In case of no difference, testing was stopped; otherwise, MANOVA testing of groups 1 and 2 was performed. If this test yielded no difference $(P$ value $>5 \%)$, results from these groups were pooled (group 1+2) and tested against group 3. In case of significant differences in MANOVA, post hoc testing (with Bonferroni correction if parameters were independent) was performed on individual parameters; accordingly all $P$ 
TABLE 1. Basic hemodynamic and left ventricle contractility parameters at 90 days follow-up

\begin{tabular}{lccc}
\hline Parameter & $\begin{array}{c}\text { Group 1 } \\
\text { (total preservation, } \mathbf{n}=\mathbf{6})\end{array}$ & $\begin{array}{c}\text { Group 2 } \\
\text { (strut-chordae preservation, } \mathbf{n}=\mathbf{8})\end{array}$ & $\begin{array}{c}\text { Group 3 } \\
\text { (total excision, } \mathbf{n}=\mathbf{8}^{*} \text { ) }\end{array}$ \\
\hline HR (beats/min) & $60.8 \pm 8.0(52.5-69.2)$ & $73.1 \pm 14.4(61.1-85.2)$ & $65.5 \pm 12.0(55.5-75.5)$ \\
LVP max (mm Hg) & $84.3 \pm 8.5(75.4-93.3)$ & $89.3 \pm 9.3(81.5-97.0)$ & $85.8 \pm 6.1(80.7-90.9)$ \\
LV EDV (mL) & $117.8 \pm 19.3(97.5-138.0)$ & $121.1 \pm 24.2(100.9-141.4)$ & $121.4 \pm 13.2(110.4-132.5)$ \\
LV ESV (mL) & $57.4 \pm 16.8(39.7-75.0)$ & $64.9 \pm 20.9(47.4-82.3)$ & $61.8 \pm 8.8(54.5-69.2)$ \\
EF (\%) & $52.2 \pm 6.9(44.9-59.4)$ & $47.5 \pm 8.4(40.5-54.5)$ & $49.1 \pm 3.8(45.9-52.3)$ \\
LV mass (g) & $145.7 \pm 12.1(133.04-158.4)$ & $157.4 \pm 33.7(129.3-185.5)$ & $166.8 \pm 26.7(144.5-189.1)$ \\
LV ${ }_{100} \mathrm{dP} / \mathrm{dt} \mathrm{max} \mathrm{(mm} \mathrm{Hg/s)}$ & $747.4 \pm 63.0(681.3-813.6)$ & $761.1 \pm 75.9(697.6-824.5)$ & $695.2 \pm 131.7(557.0-833.4)$ \\
Ees $_{100}(\mathrm{~mm} \mathrm{Hg} / \mathrm{mL})$ & $0.55 \pm 0.12(0.43-0.67)$ & $0.65 \pm 0.21(0.47-0.83)$ & $0.58 \pm 0.22(0.38-0.79)$ \\
PRSW $_{100}(\mathrm{~mm} \mathrm{Hg})$ & $34.2 \pm 11.4(22.3-46.1)$ & $32.5 \pm 12.1(22.3-42.6)$ & $27.8 \pm 15.5(13.5-42.1)$ \\
\hline
\end{tabular}

HR, Heart rate; LVP max, maximum left ventricular pressure; $L V E D V$, left ventricular end-diastolic volume; $L V E S V$, LV end-systolic volume; $E F$, ejection fraction; $L V_{100} d P / d t$ max, maximum rate of rise of $L \mathrm{PP}_{100} ; E e s_{100}$, slope of the end-systolic pressure-volume relationship; $P R S W_{100}$, slope of the stroke work and end-diastolic volume relation. Results are shown as mean \pm standard deviation ( $95 \%$ confidence intervals). Overall multivariate analysis of variance (MANOVA) for HR, LVP max, EDV, ESV, EF, and LV mass: $P=.15$; Overall MANOVA for $\mathrm{LV}_{100} \mathrm{dP} / \mathrm{dt}$ max, Ees ${ }_{100}$, and PRSW $100: P=.36$. *Group 3 included 7 animals for analysis of LV contractility parameters.

values refer to this. Stata 8.0 1984-2003 Statistics/Data Analysis package (Stata Corporation, College Station, Tex) was used for statistical analysis of data. The study sponsors had no power of decision in terms of study design; collection, analysis, and data interpretation; manuscript drafting; and decision of submission.

\section{Results}

Eleven animals died within 24 hours of surgery ( 3 pigs in group 1, 4 pigs in group 2, and 4 pigs in group 3); none of the deaths were related to LV failure. Two animals died of unknown causes on postoperative days 2 and 7. One animal died of cerebral hemorrhage on postoperative day 19. Three animals died of thrombus formation on the prosthetic valve on postoperative days 24,25 , and 45 . One long-term survivor was found to have had previous myocardial infarction at 90 days follow-up and was subsequently excluded from analysis. Causes and number of deaths were evenly distributed between groups (Fisher exact, $P=.94$ ). Consequently, the study group consisted of 22 animals during 90 (range 79-108) days follow-up (group $1, \mathrm{n}=6$; group $2, \mathrm{n}=8$; and group $3, \mathrm{n}=8$ ). There was minor nonobstructive pannus formation contralateral to the mechanical valve hinge regions in the majority of valve explants, but no cases of endocarditis.

Basic Hemodynamic and Cardiovascular Magnetic Resonance Measurements of Left Ventricular Function The hemodynamic data for all animals in each of the 3 groups are summarized in Table 1. Overall MANOVA testing showed no differences between groups $(P=.15)$. There was a slight but statistically nonsignificant tendency toward greater LV wall mass in group 3 compared with group $1+2$.

\section{Base-Apex and Short-Axis Linear Dimensions and Fractional Shortenings}

Table 2 shows mean data for LV base-apex length, shortaxis diameter, and fractional shortenings.

The central diastolic $(P<.001)$ and systolic $(P=.007)$ base-apex lengths were larger in group 3 than group $1+2$, and group 3 also had significantly greater base-apex frac-

TABLE 2. Lengths, fractional shortenings, and sphericity indices at 90 days follow-up

\begin{tabular}{lccc}
\hline Parameter & $\begin{array}{c}\text { Group 1 } \\
\text { (total preservation, n = 6) }\end{array}$ & $\begin{array}{c}\text { Group 2 } \\
\text { (strut-chordae preservation, n = 8) }\end{array}$ & $\begin{array}{c}\text { Group 3 } \\
\text { (total excision, n = 8) }\end{array}$ \\
\hline Diastolic base-apex length (mm) & $90.3 \pm 3.4(86.8-93.8)$ & $94.0 \pm 8.5(86.9-101.0)$ & $103.4 \pm 5.0(99.2-107.7)^{*}$ \\
Systolic base-apex length (mm) & $80.9 \pm 4.1(76.6-85.2)$ & $84.7 \pm 7.9(78.2-91.3)$ & $91.3 \pm 5.2(86.9-95.7) \dagger$ \\
Base-apex FS & $0.105 \pm 0.016(0.087-0.122)$ & $0.098 \pm 0.021(0.081-0.115)$ & $0.118 \pm 0.014(0.106-0.130) \ddagger$ \\
Diastolic short-axis diameter (mm) & $51.1 \pm 4.3(46.6-55.7)$ & $49.9 \pm 4.6(46.0-53.7)$ & $49.1 \pm 2.6(47.0-51.3)$ \\
Systolic short-axis diameter (mm) & $38.6 \pm 4.7(33.7-43.5)$ & $37.8 \pm 6.3(32.6-43.1)$ & $34.6 \pm 0.1 .2(33.5-35.6)$ \\
Short-axis FS & $0.247 \pm 0.036(0.209-0.285)$ & $0.245 \pm 0.072(0.185-0.306)$ & $0.294 \pm 0.040(0.262-0.328) \S$ \\
Diastolic SI & $0.32 \pm 0.06(0.26-0.38)$ & $0.28 \pm 0.08(0.22-0.35)$ & $0.20 \pm 0.03(0.18-0.23) \|$ \\
Systolic SI & $0.21 \pm 0.05(0.15-0.27)$ & $0.21 \pm 0.08(0.14-0.28)$ & $0.15 \pm 0.03(0.12-0.18) \uparrow$ \\
\hline
\end{tabular}

FS, Fractional shortening; $S$, sphericity index. Results are shown as mean \pm standard deviation (95\% confidence intervals). Group 3 versus groups 1 and 2 by multivariate analysis of variance (MANOVA): $* P<.001 . \dagger P=.007 . \ddagger P=.036 . \S P=.047 . \| P=.001 . \rrbracket[P=.034$. 
tional shortening $(P=.036)$. Also, all group 3 diastolic and systolic base-apex lengths (from apex to edges of valve) were equally and significantly increased compared with lengths of group $1+2$ (results not shown); that is, the valve did not "tilt" because of chordal severing.

Group 3 had significantly smaller diastolic $(46.1 \pm 3.2$ $\mathrm{mm}$ vs $50.3 \pm 5.8 \mathrm{~mm}, P=.018)$ and systolic $(33.7 \pm 2.0$ $\mathrm{mm}$ vs $38.9 \pm 4.9 \mathrm{~mm}, P=.010$ ) septal-lateral diameters than group $1+2$, whereas there were no differences between groups in terms of anterior-posterior diameters or mean short-axis diameters (Table 2). Also, short-axis fractional shortening was significantly greater in group 3 than group $1+2$ (Table 2, $P=.047)$.

\section{Sphericity Indices and Base-Apex/Short-Axis Indices}

Group 3 had significantly smaller diastolic $(P=.001)$ and systolic $(P=.034)$ sphericity indices (Table 2$)$ as well as greater diastolic $(2.24 \pm 0.20$ vs $1.86 \pm 0.21, P<.001)$ and systolic ( $2.62 \pm 0.32$ vs $2.25 \pm 0.31, P=.015)$ base-apex/ short-axis indices than group $1+2$.

\section{Left Ventricular Contractility Parameters}

These values are summarized in Table 1. One data set was excluded from analysis because of poor signal quality; consequently study group 3 (total excision) consisted of 7 animals. MANOVA showed no overall difference between groups $(P=.36)$. There was a slight but nonsignificant tendency toward greater $\mathrm{dP} / \mathrm{dt}$-max in group $1+2$ compared with group 3.

\section{Regional Left Ventricular Function and Geometry}

Results are shown in Figure 2. Because of the differences in base-apex lengths between groups as outlined above, we only included slices 1 through 8 for statistical analysis. Group 3 had significantly smaller end-diastolic area in slices $7(P=.010)$ and $8(P=.036)$, and also significantly smaller end-systolic area in slices $7(P=.011)$ and $8(P=.009)$ than group $1+2$ (Figure 2, $A$ and $B$ ). Myocardial thickening was not different between groups (overall MANOVA, $P=$ .32 , Figure 2, $C$ ).

\section{Discussion}

In 1956, Rushmer ${ }^{18}$ was the first to suggest a role for the mitral valve subvalvular apparatus in maintaining a synchronous and efficient LV contraction pattern. Later, Lillehei and coworkers ${ }^{19}$ applied these theories to the clinical problem of postoperative low cardiac output after MVR. Since then, several experimental ${ }^{20}$ and clinical studies ${ }^{1,5}$ have confirmed the beneficial role of the mitral subvalvular apparatus for LV function.

Our study is the first to determine the long-term impact of strut chordal preservation in a human compatible animal experimental model of MVR. This novel technique was functionally equivalent to total preservation of the subvalvular apparatus in terms of maintaining overall LV geometric and systolic function.

In contrast, total chordal resection during MVR caused significant changes of global and regional LV shape despite preserved global LV systolic function; group 3 animals developed longer and narrower ventricles compared with groups 1 and 2 . These surprising results were further elucidated by regional analysis that showed slimming to occur primarily in the basal segments, again despite evenly distributed regional myocardial thickening between groups. Regional analysis also showed that apical segment geometry was equal between all 3 groups, which may hypothetically imply that elongation predominantly took place in the basal segments.

It is noteworthy that both end-systolic and end-diastolic long-axis dimensions and long- and short-axis fractional shortening were greater in group 3 compared with groups 1 and 2. This suggests that increased longitudinal myocardial fiber stretch (because of altered preload conditions), according to the Frank-Starling mechanism, compensated for the lack of valvular-ventricular continuity in group 3 animals to maintain normal LV piston-pump function.

Although total chordal preservation MVR has been accepted as the gold standard, this method has only shown superior postoperative results when compared with MVR with preservation of the posterior leaflet and chords. ${ }^{5}$ However, the specific role of retaining primary and/or secondary chordae (ie, components of both anterior and posterior parts of the subvalvular apparatus) during MVR has not been clarified.

A number of physiologic studies have suggested different functions for primary and secondary chordae. Obadia and colleagues ${ }^{8}$ showed severe mitral insufficiency with preserved LV function when the primary chordae were cut in isolated beating pig hearts. In contrast, when secondary chordae, including strut chordae, were cut, significant LV dysfunction resulted although the mitral valve remained competent. Furthermore, Nielsen and coworkers ${ }^{9}$ showed regional LV systolic dysfunction, and recently Rodriguez and colleagues ${ }^{11}$ demonstrated both disturbed global LV function and altered LV geometry and wall thickening mechanics after cutting the secondary chordae in ovine models without MVR. In normal pig hearts, Lomholt and colleagues ${ }^{10}$ furthermore showed that secondary chordae carried a tension more than 3-fold greater than primary chordae during systole, which corroborates a principal force transmission through the strut chordae during LV ejection.

However, the evidence of differential functions of primary and secondary chordae stem from acute physiologic studies. Therefore, the effects of heterotopic transfer of the primary chordae from their insertion points on 
A

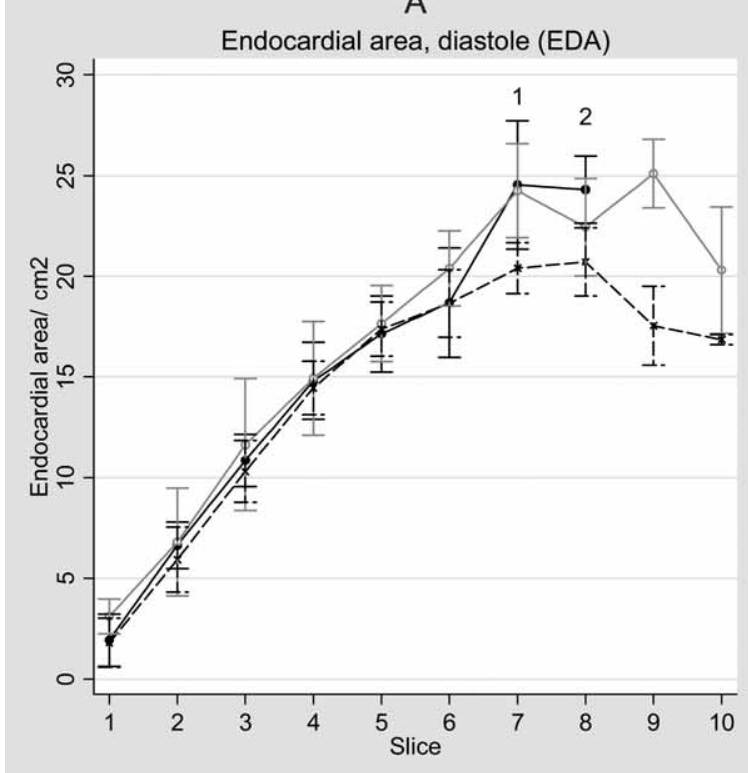

B

Endocardial area, systole (ESA)

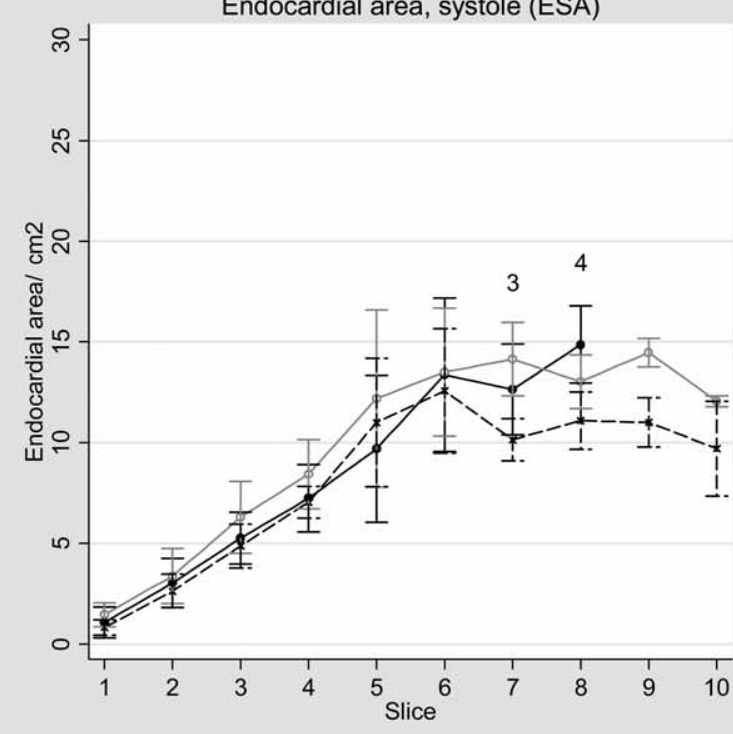

C

Myocardial thickening

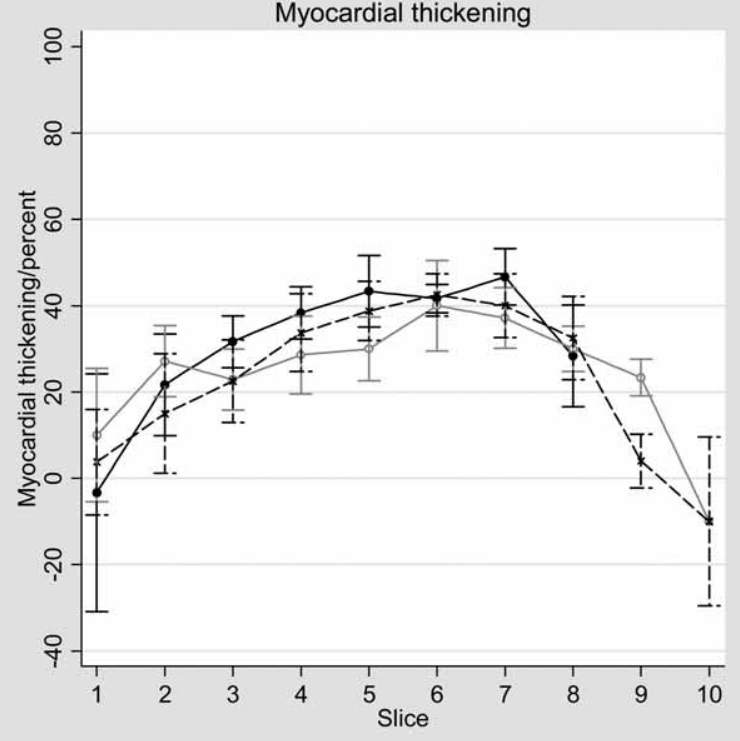

Figure 2. Regional analysis of left ventricular geometry and function. End-diastolic area (A), end-systolic area (B), and myocardial thickening (C) are shown as means with $95 \%$ confidence intervals. Group 1, solid black line; group 2, solid gray line; group 3, stippled black line. Group 3 had significantly smaller end-diastolic area in slices $7(P$ $=.010)^{1}$ and $8(P=.036)^{2}$ than groups 1 and 2 . For end-systolic area, group 3 had significantly lower values in slices $7(P=.011)^{3}$ and $8(P=.009)^{4}$ than groups 1 and 2 . Regional myocardial thickening did not differ between groups (overall MANOVA, $P=.32$ ).

the mitral valve leaflet free edges within the LV to a more taut peripheral position along the annulus are unknown. Should all chordae, with different thickness, composition, and biomechanical properties, necessarily be pre- served if they do not substantially contribute to LV function and geometry, or if they block a proper LV support by the basal chordae because of their length after transfer or stiffness? 
On the basis of these considerations, we tested an alternative and simple surgical solution to preserve the principal components of valvular-ventricular interaction and found that strut chordal preservation was equivalent to total valve preservation during MVR.

We used a long-term porcine model ${ }^{12}$ because of pigs' overall similarity to humans in terms of cardiovascular anatomy and physiology, and specifically because this species exhibit the most human comparable mitral valve apparatus. ${ }^{21,22}$ Although this study exhibited a fairly high overall mortality (18/40) during a follow-up time of 90 days, others have reported similar results with pigs. ${ }^{23,24}$ Causes of death were evenly distributed among groups, and none were related to LV or MVR failure. We therefore conclude that the possibility of bias, in terms of selection of poor animals to groups 1 and 2, and fit animals to group 3, was low.

Surprisingly there were no discernible differences in basic hemodynamic or LV contractility parameters between groups. We cannot exclude the possibility of type 2 statistical errors; however, the fairly narrow confidence intervals of these values did not suggest differences of any clinical significance and certainly not differences of the magnitude demonstrated previously by Yun and coworkers. ${ }^{25,26}$ However, these and other ${ }^{27}$ experiments have been carried out in acute settings immediately after surgery and may therefore likely reflect the "low-cardiac output syndrome" originally described in patients in the early postoperative period after MVR with excision of chordae. Clinical studies have found normalization of global LV volumes and function after MVR both with and without preservation of chordae in patients with preoperatively dilated ventricles. ${ }^{1,5}$

Our results must be interpreted in light of some study limitations. As discussed previously, the experiments were performed in normal animal hearts without the LV remodeling that may accompany chronic mitral regurgitation, and although very similar, the human and pig mitral valve apparatus has anatomic differences. Therefore, the observed results may not be directly applicable to the human mitral valve. Furthermore, the use of young animals (with $\sim 20 \mathrm{~kg}$ weight gain during 3 months follow-up) may obscure differences between treatment groups because of compensatory growth mechanisms.

\section{Conclusion}

This study is the first to determine the role of strut chordae tendineae for maintaining long-term left ventricular shape and function after MVR. We found that the novel technique of preserving only strut chordae during MVR was functionally equivalent to MVR with total chordal preservation. In contrast, total chordal resection caused significant LV lengthening and slimming despite preserved global func- tion; this was possibly the result of redistribution of contractile reserve of the LV.

Therefore, during MVR special attention must be paid to maintain the valvular-ventricular continuity through the strut chordae with the aim to preserve postoperative LV geometry and systolic function, and thus improve clinical outcome. This concept may encourage surgeons to attempt chordal-sparing techniques even in patients with severe valvular disease who would otherwise require total excision of their native mitral valve.

The extensive experimental work would have been insurmountable without the help and assistance of the technical personnel at the Institute of Clinical Medicine, Aarhus University Hospital, and Skejby Sygehus, as well as the veterinary caretaker personnel at the Påskehøjgaard Centre. In particular, Tanja Thomsen is acknowledged for her continuous aid and expertise during experiments.

\section{References}

1. David TE, Burns RJ, Bacchus CM, Druck MN. Mitral valve replacement for mitral regurgitation with and without preservation of chordae tendineae. J Thorac Cardiovasc Surg. 1984;88:718-25.

2. David TE. Mitral valve replacement with preservation of chordae tendinae: rationale and technical considerations. Ann Thorac Surg. 1986;41:680-2.

3. Moon MR, DeAnda A, Jr., Daughters GT, Ingels NB, Jr., Miller DC. Experimental evaluation of different chordal preservation methods during mitral valve replacement. Ann Thorac Surg. 1994;58:931-43.

4. Moon MR, DeAnda A, Jr., Daughters GT, Ingels NB, Miller DC. Effects of chordal disruption on regional left ventricular torsional deformation. Circulation. 1996;94(9 suppl):II143-II51.

5. Yun KL, Sintek CF, Miller DC, Pfeffer TA, Kochamba GS, Khonsari $\mathrm{S}$, et al. Randomized trial comparing partial versus complete chordalsparing mitral valve replacement: effects on left ventricular volume and function. $J$ Thorac Cardiovasc Surg. 2002;123:707-14.

6. Yacoub MH. Anatomy of the mitral valve chordae and cusps. In: Kalmonson D, editor. The mitral valve. A pluridisciplinary approach. London: Edward Arnold; 1976. p. 15-20.

7. Lam JH, Ranganathan N, Wigle ED, Silver MD. Morphology of the human mitral valve. I. Chordae tendineae: a new classification. Circulation. 1970;41:449-58.

8. Obadia JF, Casali C, Chassignolle JF, Janier M. Mitral subvalvular apparatus: different functions of primary and secondary chordae. Circulation. 1997;96:3124-8.

9. Nielsen SL, Timek TA, Green GR, Dagum P, Daughters GT, Hasenkam JM, et al. Influence of anterior mitral leaflet second-order chordae tendineae on left ventricular systolic function. Circulation. 2003;108: 406-91.

10. Lomholt M, Nielsen SL, Hansen SB, Andersen NT, Hasenkam JM. Differential tension between secondary and primary mitral chordae in an acute in-vivo porcine model. J Heart Valve Dis. 2002;11:337-45.

11. Rodriguez F, Langer F, Harrington KB, Tibayan FA, Zasio MK, Cheng A, et al. Importance of mitral valve second-order chordae for left ventricular geometry, wall thickening mechanics, and global systolic function. Circulation. 2004;110(11 suppl 1):II115-II22.

12. Smerup M, Pedersen TF, Nyboe C, Funder JA, Christensen TD, Nielsen SL, et al. A long-term porcine model for evaluation of prosthetic heart valves. Heart Surg Forum. 2004;7(4):E259-E64.

13. Cerqueira MD, Weissman NJ, Dilsizian V, Jacobs AK, Kaul S, Laskey WK, et al. Standardized myocardial segmentation and nomenclature for tomographic imaging of the heart: a statement for healthcare professionals from the Cardiac Imaging Committee of the Council on Clinical Cardiology of the American Heart Association. Circulation. 2002;105:539-42. 
14. Rajappan K, Bellenger NG, Anderson L, Pennell DJ. The role of cardiovascular magnetic resonance in heart failure. Eur J Heart Fail. 2000;2:241-52.

15. Lamas GA, Vaughan DE, Parisi AF, Pfeffer MA. Effects of left ventricular shape and captopril therapy on exercise capacity after anterior wall acute myocardial infarction. Am J Cardiol. 1989;63:1167-73.

16. Sabbah HN, Kono T, Stein PD, Mancini GB, Goldstein S. Left ventricular shape changes during the course of evolving heart failure. Am J Physiol. 1992;263(1 Pt 2):H266-70.

17. Kass DA, Midei M, Graves W, Brinker JA, Maughan WL. Use of a conductance (volume) catheter and transient inferior vena caval occlusion for rapid determination of pressure-volume relationships in man. Cathet Cardiovasc Diagn. 1988;15:192-202.

18. Rushmer R. Initial phase of ventricular systole: asynchronous contraction. Am J Physiol. 1956;184:188-94.

19. Lillehei CW, Levy MJ, Bonnabeau RC. Mitral valve replacement with preservation of papillary muscles and chordae tendinaea. $J$ Thorac Cardiovasc Surg. 1964;47:532-43.

20. Hansen DE, Cahill PD, DeCampli WM, Harrison DC, Derby GC, Mitchell RS, et al. Valvular-ventricular interaction: importance of the mitral apparatus in canine left ventricular systolic performance. Circulation. 1986;73:1310-20.
21. Walmsley R. Anatomy of human mitral valve in adult cadaver and comparative anatomy of the valve. Br Heart J. 1978;40:351-66.

22. Rijk-Zwikker GL, Delemarre BJ, Huysmans HA. Mitral valve anatomy and morphology: relevance to mitral valve replacement and valve reconstruction. J Card Surg. 1994;9(2 suppl):255-61.

23. Swan H, Meagher DM. Total body bypass in miniature pigs. Postperfusion pulmonary hypertension. J Thorac Cardiovasc Surg. 1971;61: 956-67.

24. Swan H, Piermattei DL. Technical aspects of cardiac transplantation in the pig. J Thorac Cardiovasc Surg. 1971;61:710-23.

25. Yun KL, Fann JI, Rayhill SC, Nasserbakht F, Derby GC, Handen $\mathrm{CE}$, et al. Importance of the mitral subvalvular apparatus for left ventricular segmental systolic mechanics. Circulation. 1990;82(5 suppl): IV89-104.

26. Yun KL, Rayhill SC, Niczyporuk MA, Fann JI, Zipkin RE, Derby GC, et al. Mitral valve replacement in dilated canine hearts with chronic mitral regurgitation. Importance of the mitral subvalvular apparatus. Circulation. 1991;84(5 suppl):III112-III24.

27. Gams E, Schad H, Heimisch W, Hagl S, Mendler N, Sebening F. Importance of the left ventricular subvalvular apparatus for cardiac performance. J Heart Valve Dis. 1993;2:642-5. 